\title{
MicroRNA-22 Downregulation by Atorvastatin in a Mouse Model of Cardiac Hypertrophy: a new Mechanism for Antihypertrophic Intervention
}

\author{
Yingfeng Tu ${ }^{b, c}$ Lin Wan ${ }^{a, b}$ Lihong Bu ${ }^{a, b}$ Dongliang Zhao ${ }^{a, b}$ Dandan Dong ${ }^{a, b}$ Tao Huanga,b \\ Zhen Chengd Baozhong Shen ${ }^{a, b}$ \\ aDepartment of Radiology, the Fourth Hospital of Harbin Medical University, 31 Yinhang Street, \\ Nangang District, Harbin, Heilongjiang; 'bey Laboratory of Molecular Imaging, College of Heilongjiang \\ Province, 'Department of Cardiology, the Fourth Hospital of Harbin Medical University, 31 Yinhang \\ Street, Nangang District, Harbin, Heilongjiang; dMolecular Imaging Program at Stanford, Department of \\ Radiology and Bio-X Program, Stanford University, Stanford, California
}

\section{Key Words}

Atorvastatin • MicroRNA-22 • Angiotensin II • Cardiac hypertrophy

\begin{abstract}
Background: Growing evidence shows that microRNAs (miRNAs) are involved in various cardiac processes including cardiac hypertrophy. However, the modulation of miRNA by pharmacological intervention in cardiomyocyte hypertrophy has not been disclosed yet. Methods: We constructed neonatal rat cardiomyocyte hypertrophy induced by angiotensin II stimulation and subjected to cardiomyocyte immunochemistry, qRT-PCR and immunoblotting analysis. In addition, we constructed the mouse cardiac hypertrophy using angomir-22 stimulation and demonstrated the potential antihypertrophic mechnism of atorvastatin. Results: The results showed that a collection of miRNAs were aberrantly expressed in hypertrophic cardiomyocytes induced by angiotensin II stimulation. In addition, overexpression of miR-22 was found in angiotensin II-induced hypertrophic cardiomyocytes, whereas administration of atorvastatin could reverse the upregulation of miRNA-22 nearly back to the control level. Furthermore, up-regulation of miRNA-22 in cardiomyocytes in vitro and in vivo could induce cardiac hypertrophy, which could suppress the protein level of phosphatase and tensin homolog deleted on chromosome ten (PTEN). Concomitantly, the production of ANP, BNP and $\beta-\mathrm{MHC}$ was enhanced and cardiomyocyte size was increased. However, atorvastatin could markedly knockdown miRNA-22 expression and reverse these changes in cardiomyocytes. These results suggest that the contribution of atrovastatin to cardiomyocyte hypertrophy may be involved in downregulation of miRNA-22 expression, which modulates the activity of PTEN in cardiomyocyte hypertrophy. Conclusion: This study demonstrates for the first
\end{abstract}

Dr. Baozhong Shen and

PHD. Zhen Cheng
Department of Radiology, the Fourth Hospital of Harbin Medical University

31 Yinhang Street, Nangang District, Harbin, Heilongjiang (PR China)

and Molecular Imaging Program at Stanford, Department of Radiology and Bio-X

Program, Stanford University, Stanford, California (USA)

E-Mail shenbzh@vip.sina.com and E-Mail zcheng@stanford.edu 
Tu/Wan/Bu/Zhao/Dong/Huang/Cheng/Shen: MicroRNA-22 Downregulation by Atorvastatin in Mouse Cardiac Hypertrophy

time the modulation of miRNA-22 can be achieved by pharmacological intervention. The data generated from this study provides a rationale for the development of miRNA-based strategies for antihypertrophic treatment.

Copyright $@ 2013$ S. Karger AG, Basel

\section{Introduction}

Cardiac hypertrophy is a common response to a variety of physiological as well as pathophysiological stimuli. The typical features of cardiac hypertrophy are reactivation of fetal genes, reorganization of sarcomere, and enlargement of cardiomyocyte size [1]. Although physiological hypertrophy shows an enhancement of cardiac function, prolonged cardiac hypertrophy will eventually lead to heart failure or sudden death by activating intracellular signaling pathways and transcriptional mediators in cardiac myocytes [2]. Because of the grave consequence of cardiac hypertrophy, many scientific works endeavor to disclose the underlying mechanisms of pathological hypertrophy and reverse its deleterious aspect. There have been major advances in the identification of cardiac autonomous and endocrine/paracrine signaling pathways involved in cardiac hypertrophy process, but the overall complexity of hypertrophic remodeling suggests that additional regulatory mechanisms remain poorly understood.

Current treatments for cardiac hypertrophy are limited to vasodilators or afterload reducers, but few other therapies are directed at the myocardial process. The HMG-CoA reductase inhibitors are widely prescribed cholesterol-lowering agents that decrease the mortality and morbidity in patients with atherosclerotic cardiovascular disease. Previous investigations have suggested that statins may be involved in antihypertrophic role through multiple mechanisms. For example, atorvastatin prevents the development of cardiac hypertrophy partly through reducing active Ras and p44/42 MAPK [3]. Hypoxia in neonatal cardiomyocytes increases ROS and myocardin expression to cause cardiomyocyte hypertrophy, which can be prevented by atorvastatin by suppressing ROS and myocardin expression [4]. Laufs and colleagues proved that statins inhibit MLC-2 and ANF expression in hypertensive rats, again accompanied by a decrease in GTP binding activity of RhoA and Rac1 [5]. Rodrigues et al. demonstrated that statins inhibit several angiotensin II (Ang II)activated intracellular signaling systems, including p38-MAPK and ROCK, which regulate the AngII/Smad pathway and related profibrotic factors [6]. PTEN, as a negative regulator of phosphatidylinositol-3 kinase (PI3K)-AKT pathways, was recently found to be decreased in hypertrophic cardiomyopathy and to be involved in a wide variety of hypertrophic adaptive responses in cardiomyocytes [7]. Researchers transfected PTEN into neonatal rat cardiomyocytes and found that it inhibits Ang II-induced cardiac myocytes hypertrophy, suggesting that PTEN is involved in negative modulation of cardiac myocytes hypertrophy [8]. Planavila et al. elucidated a new potential antihypertrophic effect of atorvastatin, which can inhibit activation of the Akt/GSK-3 $\beta$ hypertrophic pathway by regulating PTEN activation [9].

MicroRNAs (miRNAs) are a class of small non-coding RNAs that mediate posttranscriptional gene silencing. Growing evidences show that miRNAs play pivotal roles in diverse aspects of heart diseases, including myocardial ischemia $[10,11]$, apoptosis $[12$, 13], arrhythmia [14], cardiac fibrosis [15], and heart failure [16, 17]. Notably, evidence from recent studies has shown that miRNAs are involved in regulating cardiomyocyte cell size, cardiac growth and fibrosis in cardiac hypertrophy $[18,19]$. A recent study demonstrated that miR-21 regulates MMP-2 expression in cardiac fibroblasts of the myocardial infarct zone through a PTEN pathway [20]. And Xu et al. proved that attenuation of miR-22 in rat cardiomyocytes efficiently protected from hypertrophic effects through derepressing PTEN [21]. The previous studies suggest that there is a therapeutic potential for miRNAs treatment of cardiovascular disease. Antagomirs are now used as a means to constitutively inhibit 
Tu/Wan/Bu/Zhao/Dong/Huang/Cheng/Shen: MicroRNA-22 Downregulation by Atorvastatin in Mouse Cardiac Hypertrophy

the activity of specific miRNAs [22]. However, it remains a challenge to use antagomir as a promising treatment in clinical trials because it is hard to deliver and the fact that specificity of targeting miRNA is difficult to realize.

The aims of the present study are to determine the role of miRNAs in Ang II-induced cardiac hypertrophy. In particular, we put forward that specific miRNA can be modulated by pharmacological intervention. In this study, we focused on the interaction between miR22 and atorvastatin in Ang II-induced cardiac hypertrophy. To this end, we evaluated the antihypertrophic effects of atorvastatin and disclosed the underlying mechanisms, which was involved in regulation of miR-22 expression in cardiomyocyte hypertrophy in vitro and in vivo.

\section{Materials and Methods}

\section{Animal care}

Eight-week-old male Kunming mice (25-28g), which were supplied by the Medical Experimental Animal Center of Harbin Medical University, China. The mice were fed under standard animal room conditions (temperature $21 \pm 1^{\circ} \mathrm{C}$; humidity 55-60\%). Food and water were freely available throughout the experiments. All procedures were approved by the ethical committee of Harbin Medical University, China and were in accordance with the National Institutes of Health Guidelines for the care and use of experimental animals (NIH Publication No. 85-23, revised 1996).

\section{Cardiomyocyte culture and cell models for hypertrophy}

The myocyte dissociation procedure was similar to that described previously [23]. In brief, neonatal rat cardiomyocytes were isolated and cultured from the ventricles of 2-day-old Sprague-Dawley rats. The ventricles were excised, washed and cut into small pieces in serum free DMEM medium, then digested with a $0.25 \%$ solution of trypsin in a carbon dioxide incubator to keep the reaction temperature at $37^{\circ} \mathrm{C}$. After dissociation, the cells were subjected to centrifugation, followed by differential preplating to enrich cardiomyocytes and deplete nonmyocytes. And then, the purified cardiomyocytes were diluted to $1 \times 10^{6}$ cells $/ \mathrm{ml}$ and plated on flasks in DMEM supplemented with $10 \%$ fetal bovine serum (FBS). Having been cultured for $48 \mathrm{~h}$, the medium was replaced with a serum free maintenance medium and incubated for another $24 \mathrm{~h}$. Then the culture medium was changed to serum-free DMEM and cells were pretreated with atorvastatin in dosage $(10 \mu \mathrm{mol} / \mathrm{L})$ or phosphate buffer saline (PBS) for $30 \mathrm{~min}$ and subsequently stimulated with $1 \mu \mathrm{mol} / \mathrm{L}$ Ang II (Sigma, USA) for $48 \mathrm{~h}$.

\section{Quantitative reverse transcription-PCR ( $q R T-P C R$ )}

Total RNA samples from cultured cardiomyocytes and cardiac tissues were isolated using Trizol reagent (Invitrogen, USA) according to manufacturer's protocols. Total RNA $(0.5 \mu \mathrm{g})$ was then reverse transcribed using High-Capacity cDNA Reverse Transcription Kit (Applied Biosystems, USA) to obtain cDNA. The SYBR Green PCR Master Mix Kit (Applied Biosystems, USA) was used in qRT-PCR to quantify the RNA levels of miR-1, miR-9, miR-15b, miR-21, miR-22, miR-23a, miR-24, miR-26a, miR-27, miR-133, miR-199b, miR-208, miR-214 and miR-499 using SYBR Green PCR Master Mix Kit, with U6 as an internal control. The qRT-PCR was performed on 7500 FAST Real-Time PCR System (Applied Biosystems, USA) for 40 cycles. The hypertrophic markers such as ANP, BNP and $\beta$-MHC in cardiomyocytes were detected also using qRT-PCR analysis, with GAPDH as an internal control. The sequences of primers were in Table 1 and Table 2.

\section{Transfection of miR-22 mimic into cultured cardiomyocytes}

The cells were seeded in antibiotic-free medium for $24 \mathrm{~h}$ prior to transfection. For the miR-22 upregulation, the cells were transfected with miR-22 mimic (GenePharma Co. Ltd.) using Lipofectamine 2000 (Invitrogen, USA). Transfection complexes were added to medium at final oligonucleotide concentration of $50 \mathrm{nM}$. For the miR-22 knockdown, the cardiomyocytes were pretreated with atorvastatin in dosage $(10 \mu \mathrm{mol} / \mathrm{L})$ before transfection. The culture medium was replaced $4 \mathrm{~h}$ post-transfection with the regular culture medium for another $24 \mathrm{~h}$. MiRNA transfection efficiency was proved by qRT-PCR. 


\begin{tabular}{|c|c|c|}
\hline \multirow{2}{*}{$\begin{array}{l}\text { Cellular Physiolosy } \\
\text { and Biochemistry }\end{array}$} & \multicolumn{2}{|c|}{ Cell Physiol Biochem 2013;31:997-1008 } \\
\hline & $\begin{array}{l}\text { DOI: 10.1159/000350117 } \\
\text { Published onlIne: July 0Z, } 2013\end{array}$ & $\begin{array}{l}\text { (c) } 2013 \text { S. Karger AG, Basel } \\
\text { www.karger.com/cpb }\end{array}$ \\
\hline
\end{tabular}

Table 1. Primers for reverse-transcription of miRNAs

\begin{tabular}{ll}
\hline miRnAs & Primers for reverse-transcription \\
\hline miR-1 & 5'-GTCGTATCCAGTGCAGGGTCCGAGGTATTCGCACTGGATACGACCTGCTC-3' \\
miR-9 & 5'-GTCGTATCCAGTGCGTGTCGTGGAGTCGGCAATTGCACTGGATACGACTCATAC-3' \\
miR-15b & 5'-GTCGTATCCAGTGGGTGTCGTGGAGTCGGCAATTGCACTGGATACGACTGTAAAC-3' \\
miR-21 & 5'-GTCGTATCCAGTGCAGGGTCCGAGGTATTCGCACTGGATACGACTCAACA-3' \\
miR-22 & 5'-GTTGGCTCGGTGCAGGGCCTGAGGTATTCGCACCAGAGCCAACACAGTTC-3' \\
miR-23a & 5'-GTCGTATCCAGTGCGTGTCGTGGAGTCGGCAATTGCACTGGATACGACGGAAATC-3' \\
miR-24 & 5'-GTCGTATCCAGTGCGTGTCGTGGAGTCGGCAATTGCACTGGATACGACCTGTTCC-3' \\
miR-26a & 5'-GTCGTATCCAGTGCAGGGTCCGAGGTATTCGCACTGGATACGACAGCCTAT-3' \\
miR-27a & 5'-GTCGTATCCAGTGCGTGTCGTGGAGTCGGCAATTGCACTGGATACGACGCGGAA-3' \\
miR-133a & 5'-GTCGTATCCAGTGCAGGGTCCGAGGTATTCGCACTGGATACGACACAGCT-3' \\
miR-199b & 5'-GTCGTATCCAGTGCGTGTCGTGGAGTCGGCAATTGCACTGGATACGACTAACCAA-3' \\
miR-208a & 5'-GTCGTATCCAGTGCGTGTCGTGGAGTCGGCAATTGCACTGGATACGACGTATAAC-3' \\
miR-214 & 5'-GTCGTATCCAGTGCGTGTCGTGGAGTCGGCAATTGCACTGGATACGACCTGCCT- 3' \\
miR-499 & 5'-GTCGTATCCAGTGCGTGTCGTGGAGTCGGCAATTGCACTGGATACGACAAACAT-3' \\
U6 & 5'-CGCTTCACGAATTTGCGTGTCAT-3' \\
\hline
\end{tabular}

Table 2. Primers for real-time PCR

\begin{tabular}{lll}
\hline \multirow{2}{*}{ MiRNA } & \multicolumn{2}{c}{ Primers for real-time PCR } \\
& Forward primer & Reverse primer \\
\hline miR-1 & 5'-CTGCAGCTACGTCTCAGAGC-3' & 5'-GTGCAGGGTCCGAGGT-3' \\
miR-9 & 5'-GGGGTCTTTGGTTATCTAG-3' & 5'-ATCCAGTGCGTGTCGTGGA-3' \\
miR-15b & 5'-GGGTAGCAGCACATCATG-3' & 5'-TATCCAGTGCGTGTCGTG-3' \\
miR-21 & 5'-GGGGTAGCTTATCAGACTG-3' & 5'-TGGAGTCGGCAATTGCACTG-3' \\
miR-22 & 5'-CGGCGGAAGCTGCCAGTTGAA-3' & 5'-GTGCAGGGTCCGAGGT-3'. \\
miR-23a & 5'-GGATCACATTGCCAGGGAT-3' & 5'-CAGTGCGTGTCGTGGAGT-3' \\
miR-24 & 5'-GGGGTGGCTCAGTTCAGCA-3' & 5'-CAGTGCGTGTCGTGGAGTC-3' \\
miR-26a & 5'-GCGGTTCAAGTAATCCAGG-3' & 5'-TGTCGTGGAGTCGGCAATT-3' \\
miR-27a & 5'-GGGGTTCACAGTGGCTAA-3' & 5'-AGTGCGTGTCGTGGAGTC-3' \\
miR-133a & 5'-CTGCATTGGTCCCCTTCAAC-3' & 5'-GTGCAGGGTCCGAGGT-3' \\
miR-199b & 5'-GGGACAGTAGTCTGCACAT-3' & 5'-TGTCGTGGAGTCGGCAATT-3' \\
miR-208a & 5'-GCTTGAGCTTTTGGCCCG-3' & 5'-CGTGGAGTCGGCAATTGC-3' \\
miR-214 & 5'-GGGGACAGCAGGCACAGAC-3' & 5'-AGTGCGTGTCGTGGAGTCG-3' \\
miR-499 & 5'-GGGGTTAAGACTTGCAGTG-3' & 5'-CAGTGCGTGTCGTGGAGT-3' \\
U6 & 5'-GCTTCGGCACATATACTAAAAT-3' & 5'-CGCTTCACGAATTTGCGTGTCAT-3' \\
\hline
\end{tabular}

Cardiomyocyte immunochemistry and cell surface area analysis

Cardiomyocytes were plated at a density of $5 \times 10^{4} \mathrm{cells} / \mathrm{ml}$ to obtain individual cells. After Ang II treatment for $48 \mathrm{~h}$, the cells were fixed with $4 \%$ paraformaldehyde for 30 minutes and permeabilized with $0.1 \%$ Triton X-100 in PBS, followed by blocking with $5 \%$ goat serum for $1 \mathrm{~h}$ at room temperature. The cells were incubated with monoclonal antibody against sarcomeric $\alpha$-actinin (1:200 dilution, Sigma) at $4^{\circ} \mathrm{C}$ overnight. Nuclear staining was performed with 4', 6-diamidino-2-phenylindole (DAPI) (1:10 dilution, Sigma). The cell picture was captured with laser scanning confocal microscopy (FV300, Olympus, Japan) and the cell surface area was determined with ImagePro Plus software (version 6.0, Media Cybernetics).

\section{Animal experiments}

The male Kunming mice $(25 \sim 28 \mathrm{~g})$ were maintained under standard illumination and temperature for 5 days before study began. The animals were randomly distributed into three groups as follows: control group (control, $n=5$ ), angomir-22 stimulation mice without atorvastatin pre-treatment (angomir-22, n=5) 
Tu/Wan/Bu/Zhao/Dong/Huang/Cheng/Shen: MicroRNA-22 Downregulation by Atorvastatin in Mouse Cardiac Hypertrophy

and angomir-22 stimulation mice with atorvastatin pre-treatment (angomir-22+ATV, n=5). One week before the surgical procedure, the mice received 7 days pre-treatment with atrovastatin $5 \mathrm{mg} / \mathrm{kg} /$ day (angomir$22+\mathrm{ATV}$ group) or $0.9 \%$ salt solution (control group and angomir-22 group) by oral gavage. After 7 days pretreatment with atrovastatin or salt solution, the mice were anesthetized with pentobarbital sodium (50 mg/ $\mathrm{kg}$ ip), then a polyethylene tube was intubated orally for artificial respiration (UGO Bsile S.R.L. Biological Research Apparatus, Italy). An incision $(1.0 \mathrm{~cm})$ was made on the back of each animal between the shoulder blades, and a micro-osmotic pump (1007D, Alzet Corp) containing angomir-22 $(80 \mathrm{mg} / \mathrm{kg}$ body weight dissolved in $0.9 \%$ sodium chloride solution) was inserted into the infrascapular s.c. tissue as previously described in angomir-22 group [24]. The control group were administrated with $0.9 \%$ sodium chloride solution via the micro-osmotic pump. After surgery the mice received food and water ad libitum. Hearts were collected 2 weeks after operation. The heart weight/body weight $(\mathrm{HW} / \mathrm{BW})$ ratio was calculated and the heart samples were frozen in liquid nitrogen and then stored at $-80{ }^{\circ} \mathrm{C}$.

\section{Echocardiography}

Two week after angomir-22 treatment, mice were anesthetized with $2.5 \%$ (vol/vol) isoflurane and placed on the experimental platform. Transthoracic echocardiography was performed using a highresolution in vivo ultrasound imaging system (Vevo 770 Micro-Ultrasound, Visualsonics Inc., Toronto, Canada) with a $30-\mathrm{MHz}$ phased-array transducer. Two-dimensional guided $\mathrm{M}$-mode tracings were recorded from the parasternal long-axis view at the mid papillary muscle level [25]. When the picture was stabilized, ventricular parameters including heart rate (HR), left ventricular end-diastolic dimensions (LVEDD), left ventricular end-systolic dimensions (LVESD), diastolic interventricular septal thickness (IVSTD), interventricular septal thickness in systole (IVSTS), posterior wall thickness in diastole (PWTD), and posterior wall thickness in systole (PWTS) were measured. All of the measurements were made from more than three beats and averaged. After functional measurement, mice were killed and the hearts were collected in $4 \%$ paraformaldehyde or liquid nitrogen for use.

\section{Histology}

The left ventricle was fixed in $4 \%$ paraformaldehyde for $48 \mathrm{~h}$ and embedded with paraffin. Crosssectional slices along the minor axis were obtained with a microtome and then stained using Mayer's Hematoxylin and Eosin. Cardiomyocyte hypertrophy was quantitated by measuring the diameter of 100 randomly sectioned (transverse) cardiomyocytes per microscopic field. Masson's trichrome staining was used to evaluate collagen deposition. Sections were imaged at 200×magnification by bright-field microscopy (IX71 Olympus, Japan). All quantitative evaluations were carried out by ImagePro Plus software (version 6.0, Media Cybernetics).

\section{Western blot analysis}

Briefly, the protein concentrations were determined with a bicinchoninic acid protein assay kit using bovine serum albumin as the standard. Equal amounts of protein $(100 \mu \mathrm{g})$ were fractionated by SDS-PAGE and blotted to PVDF membrane (Millipore, Bedford, MA). Membranes were blocked for $1 \mathrm{~h}$ using $5 \%$ non-fat milk in Tris-buffered saline with Tween (TBST), then probed overnight at $4{ }^{\circ} \mathrm{C}$ with the following primary antibodies: PTEN (1:1000 dilution, Cell Signaling Technology), ANP (1:200 dilution, Abcam), BNP (1:200 dilution, Abcam), $\beta$-MHC (1:1000 dilution, Cell Signaling Technology), and anti-GADPH (1:5000 dilution; Cell Signaling), all in 5\% milk TBST. Following incubation with the primary antibodies, membranes were incubated with secondary antibody (1:8000 dilution, Alexa Fluor® 700 goat anti-mouse IgG (H+L) or Alexa Fluor® 800 goat anti-rabbit IgG $(\mathrm{H}+\mathrm{L})$, Invitrogen) in PBS at room temperature for $1 \mathrm{~h}$. Western blot bands were captured by using the Odyssey Infrared Imaging System (LI-COR Biosciences, Lincoln, NE, USA) and quantified with Odyssey v1.2 software (LI-COR Biosciences, Lincoln, NE, USA) by measuring the band intensity (area×OD) in each group and normalizing to GAPDH as an internal control. Unless otherwise stated, western blot experiments were repeated three times.

\section{Statistical analysis}

All quantitative data are expressed as the mean \pm SEM and analysed by SPSS 13.0 software. Two-tailed unpaired Student's t-tests and one-way ANOVA were used for statistical evaluation of the data. Differences were considered as statistically significant when $p<0.05$. 


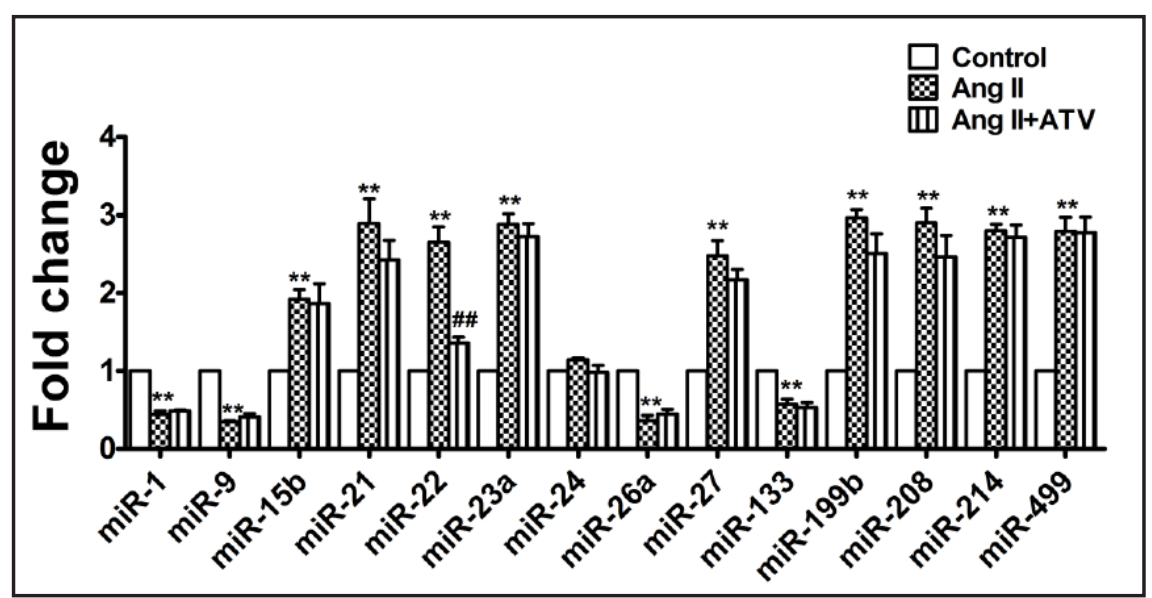

Fig. 1. Using qRT-PCR analysis, a collection of miRNAs were dynamically regulated in Ang II-induced cardiomyocyte hypertrophy. Compared with control, miR-1, miR-9, miR-26a and miR-133a were downregulated, while miR-15b, miR-21, miR-22, miR23a, miR-24, miR-27, miR-199a, miR-208, miR-214, and miR-499 were substantially upregulated in Ang II-induced cardiomyocyte hypertrophy. However, the upregulation of miRNA-22 was counteracted by co-treatment of atorvastatin. Data are expressed as mean \pm SEM. ${ }^{* *} p<0.01$ vs control group; \#\#p<0.01 vs Ang II group; $\mathrm{n}=3$ independent experiments for each condition.

\section{Results}

Effect of atorvastatin on miRNAs expression in Ang II-induced cardiomyocytes hypertrophy

As previously reported, there were a collection of miRNAs abnormally expressed in hypertrophic heart in response to Ang II or isoproterenol-induced cardiac hypertrophy, such as miR-1, miR-9, miR-15b, miR-21, miR-22, miR-23a, miR-24, miR-26, miR-27, miR133a, miR-199a , miR-208, miR-214 and miR-221 [21, 26]. In order to investigate whether these miRNAs are involved in the antihypertrophic effect of atorvastatin in Ang II-induced cardiomyocytes hypertrophy, rat cardiomyocytes were challenged with atorvastatin (10 $\mu \mathrm{mol} / \mathrm{L})$ and Ang II $(1 \mu \mathrm{mol} / \mathrm{L})$ stated in methods section. Then the expression level of aforesaid miRNAs was analyzed by qRT-PCR. Compared with control, the expression of miR1, miR-9, miR-26a and miR-133a was substantially downregulated $(p<0.01)$ (Fig. 1), while the expression of miR-21, miR-22, miR-23a, miR-27, miR-199a, miR-208, miR-214 and miR-499 was upregulated in Ang II-induced cardiomyocytes hypertrophy $(p<0.01)$ (Fig. 1). However, the upregulation of miRNA-22 was counteracted by co-treatment of atorvastatin $(p<0.01)$ (Fig. 1), indicating miRNA-22 may be involved in the antihypertrophic role of atorvastatin. In addition, the upregulation of miR-21 induced by Ang II could be mildly counteracted by co-intervention of atorvastatin (Fig. 1). Accordingly, the surface area of Ang II-stimulated myocytes was increased significantly relative to normal myocytes $(2.0022 \pm 0.04215$ vs $1.0000 \pm 0.02339, p<0.01$ ), while atorvastatin reversed the change obviously at a dosage of $10 \mu \mathrm{mol} / \mathrm{L}(1.3118 \pm 0.01964$ vs $2.0022 \pm 0.04215, p<0.01$ ) (Fig. $2 \mathrm{~A}$ and $2 \mathrm{~B}$ ). Concomitantly, the production of ANP, BNP and $\beta$-MHC was enhanced by Ang II treatment and atorvastatin could obviously reverse the increase of these proteins expression in vitro $(p<0.01)$ (Fig. 2C).

Atorvastatin markedly inhibits cardiomyocyte hypertrophy induced by upregulation of miRNA-22 in vitro

To disclose the effect of miRNA-22 in cardiomyocyte hypertrophy, we treated the cardiomyocytes with miRNA-22 mimic. The synthesized miRNA-22 mimic was transfected into cardiomyocytes for $48 \mathrm{~h}$ as described previously, which has been confirmed to have the ability of mimicing the effects of endogenous miRNA-22 on cardiomyocyte hypertrophy 
Fig. 2. Ang II-induced cardiomyocyte hypertrophy was attenuated by atorvastatin. (A) Cardiomyocytes were stained for $\alpha$-actinin proteins and DAPI (Original magnification, $\times 200$ ). (B) Quantification of cell surface area from conditions in A using immunochemistry and cell surface area analysis, $\mathrm{n}=100$ independent experiments for each condition. (C) Ang II-induced cardiomyocyte hypertrophy significantly increased ANP, BNP and $\beta$-MHC expression level, which could be attenuated by atorvastatin pre-treatment in cardiomyocytes, $n=3$ independent experiments for each condition. Data are expressed as mean \pm SEM. ${ }^{* *} p<0.01$ vs control; \#\#p $<0.01$ vs Ang II group.

Fig. 3. Atorvastatin could inhibit cardiomyocyte hypertrophy induced by up-regulation of miR-22. (A) Cardiomyocytes were stained for $\alpha$-actinin proteins and DAPI (Original magnification, $\times 200$ ). (B) Quantification of cell surface area from conditions in A using immunochemistry and cell surface area analysis. (C) ANP, BNP and $\beta$-MHC expression level in different groups by qRT-PCR analysis. (D) MiR22 expression level in different groups via qRT-PCR analysis. (E) Protein levels of PTEN. (F-H) Protein levels of ANP, BNP and $\beta$-MHC, $n=3$ independent experiments for each condition. Data are expressed as mean \pm SEM. ${ }^{* *} p<0.01$ vs control, \#\#p<0.01 vs Ang II group.
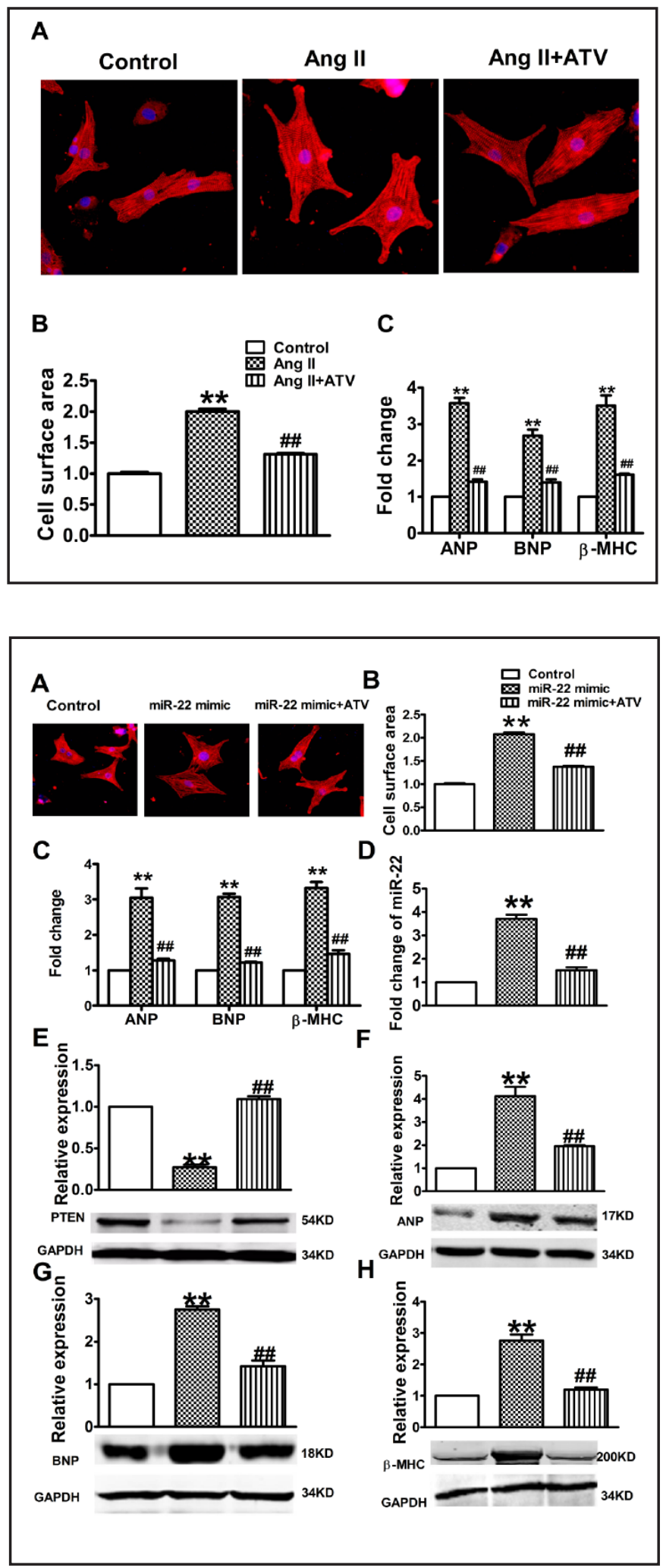

[21]. We excitingly observed that upregulation of miRNA-22 in cardiomyocytes markedly increased cell area of cardiomyocytes using cardiomyocyte immunochemistry and cell surface area analysis, and atorvastatin could abrogate the effects of miRNA-22 mimic $(p<0.01)$ (Fig. 3A and 3B). Using qRT-PCR analysis, we also found that overexpression of 


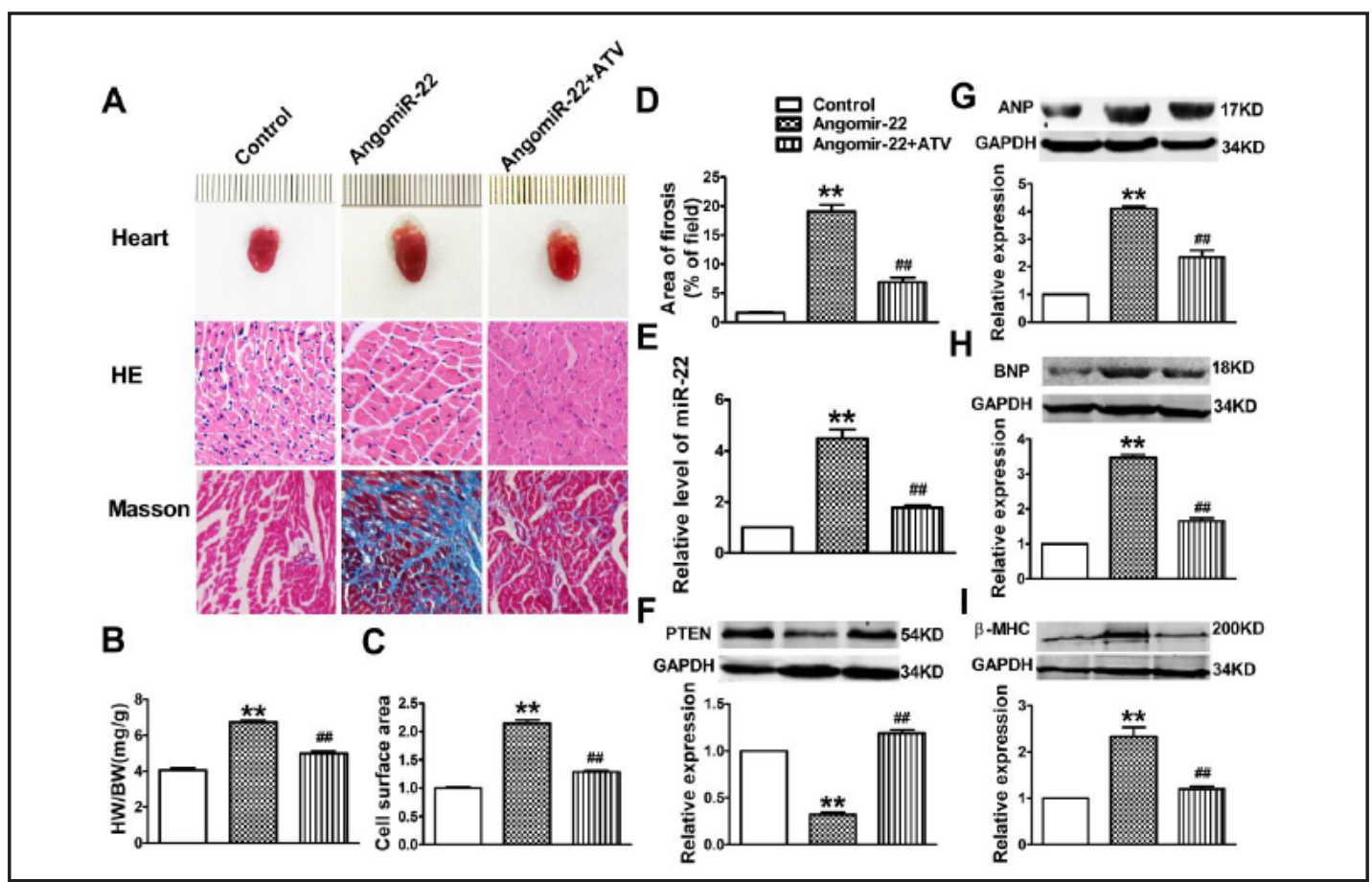

Fig. 4. (A) Gross hearts in the top row, heart sections stained with hematoxylin and eosin in the middle row and Masson's trichrome staining in the bottom row. (B) Heart weight to body weight ratios (HW/BW) in different groups, $n=5$ independent experiments for each condition. (C) The cross-sectional areas analyzed in different conditions, $n=100$ independent experiments for each condition. (D) Collagen deposition was quantified with an automated image analyzer and expressed as percentage of tissue area, $\mathrm{n}=5$ independent experiments for each condition. (E) MiR-22 expression level in different groups via qRT-PCR analysis, $\mathrm{n}=3$ independent experiments for each condition. (F-I) Protein levels of PTEN, ANP, BNP and $\beta-M H C, n=3$ independent experiments for each condition. Data are expressed as mean \pm SEM. ${ }^{* *} p<0.01$ vs control, \#\#p $<0.01$ vs Ang II group.

miRNA-22 in cardiomycytes significantly increased ANP, BNP, and $\beta$-MHC expression level in cardiomyocytes, while atorvastatin could reverse these overexpression $(p<0.01)$ (Fig. 3C). Concomitantly, we discovered that miRNA- 22 mimic could significantly upregulate miRNA-22 level by 3-fold after transfection of miRNA-22 mimic for $48 \mathrm{~h}$, which was abolished by cotreatment of atorvastatin $(p<0.01)$ (Fig. 3D). These results indicated the possible involvement of miRNA-22 in promoting cardiac hypertrophy, and atorvastatin could effectively suppress cardiomyocyte hypertrophy induced by upregulation of miRNA-22 in cardiomyocytes.

\section{PTEN is a potential antihypertrophic target of atorvastatin via inhibiting miR-22} expression in cardiomyocytes

PTEN, as a known antagonist to PI3K signaling, can serve as a critical determinant of cardiomyocyte growth. Overexpression of a catalytically inactive PTEN mutant could induce cardiomyocyte hypertrophy, with increased cell surface area, protein synthesis, and ANP expression [8]. This hypertrophy was accompanied by an increase in Akt activity and improved cell viability. We investigated whether PTEN expression was modulated by overexpression of miR-22 and whether atrovastatin could down-regulate PTEN expression by inhibiting miR-22 in vitro. The western blotting results showed that overexpression of miR-22 in cardiomyocytes significantly down-regulated PTEN expression level, while atrovastatin could inhibit endogenous miR-22 expression and markedly increased PTEN protein level $(p<0.01)$ (Fig. 3E). Concomitantly, we discovered that miRNA-22 mimic could significantly upregulate hypertrophic markers ANP, BNP and $\beta$-MHC after transfection of 
Fig. 5. Representative M-mode echocardiographs from control, angomir-22, angomir-22+ATV. LVEDD, left ventricular enddiastolic dimension; LVESD, left ventricular end-systolic dimension; IVSTD, interventricular septal thickness in diastole; IVSTS, interventricular septal thickness in systole; PWTD, posterior wall thickness in diastole; PWTS, posterior wall thickness in systole. Data are expressed as mean \pm SEM. ${ }^{*} p<0.05, * * p<0.01$ vs control, ${ }^{\#} p<0.05,{ }^{\# \#} p<0.01$ vs Ang II group.

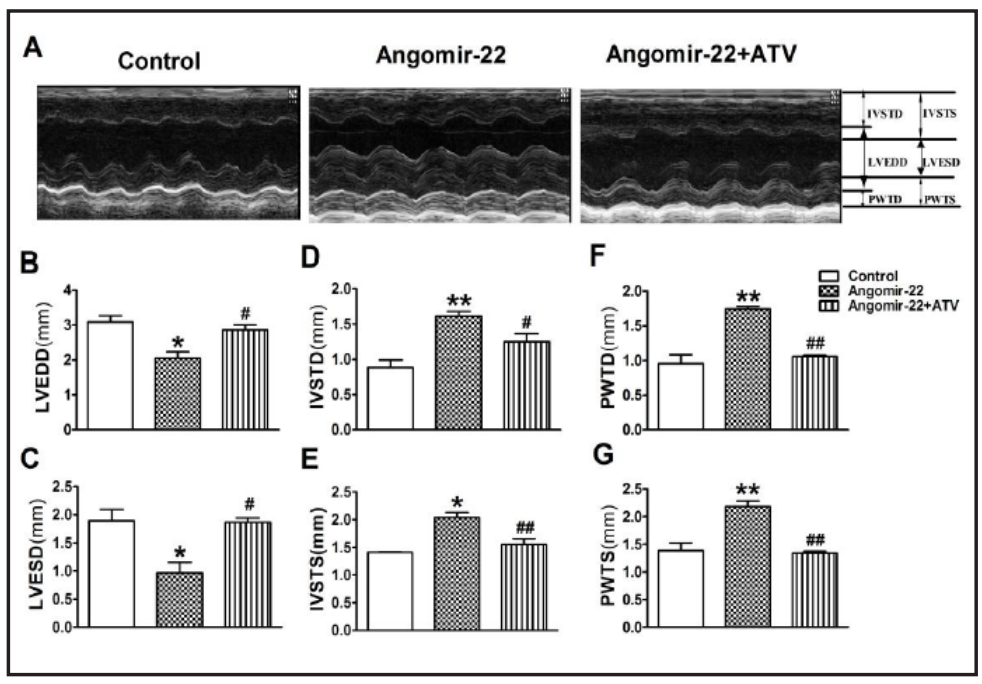

miRNA-22 mimic for $48 \mathrm{~h}$, which was abolished by co-treatment of atorvastatin $(p<0.01)$ (Fig. 3F-3H).

Atorvastatin significantly inhibits mouse cardiac hypertrophy induced by angomir-22 stimulation

We then turned to investigate if the beneficial effects of atorvastatin exist in in vivo conditions. To determine the antihypertrophic role of atorvastatin in miR-22 upregulationinduced cardiac hypertrophy, we overexpressed miR-22 specifically in the mouse heart using angomir-22 treatment stated in the methods section. As shown in Fig. 4A, angomir-22 induced cardiac hypertrophy and fibrosis could be successfully inhibited by atorvastatin pre-treatment. Atorvastatin inhibited angomir-22 induced hypertrophy revealed by heart weight/body weight ratio ( $p<0.01)$ (Fig. 4B), cross-sectional areas $(p<0.01)$ (Fig. 4C), area of fibrosis $(p<0.01)$ (Fig. 4D), expression levels of PTEN ( $p<0.01)$ (Fig. 4E) and hypertrophic markers including ANP, BNP, and $\beta$-MHC $(p<0.01)$ (Fig. 4G -4I).

Cardiac function was examined using echocardiography 2 weeks after angomir-22 transfection. Analysis of cardiac function by M-mode echocardiography revealed that mouse hearts treated with angomir-22 displayed thickening of the ventricular walls, while atorvastatin could reverse the change (Fig. 5A). Echocardiography examination showed that in angomir-22 treatment mouse hearts LVEDD and LVESD were significantly decreased, while IVSTD, IVSTS, PWTD and PWTS were markedly increased, indicating the cardiac hypertrophy $(p<0.05)$ (Fig. 5B-5G). However, atorvastatin played antihypertrophic role in vivo and reversed angomir-22 induced cardiac hypertrophy ( $p<0.05)$ (Fig. 5B-5G).

\section{Discussion}

Several novel findings of present study are summarized as follows. First, we showed that miR-22 is involved in Ang II-induced cardiac hypertrophy. Up-regulation of miR-22 via transfection of miR-22 mimic into cultured cardiomyocytes can induce cardiomyocyte hypertrophy in vitro. Second, we demonstrated that miR-22 level was downregulated by the HCM-CoA reductase inhibitor atorvastatin. Third, HCM-CoA reductase inhibitor atorvastatin produces protective effects against cardiac hypertrophy, possibly partially by reducing miR-22 expression, which might be responsible for the down-regulation of PTEN during hypertrophy. These findings not only help us understand the mechanisms underlying the antihypertrophic effects of HCM-CoA reductase inhibitor but also advance our view of miRNAs that may serve as potential pharmacological intervention targets. 
Pharmacological agents blocking or inhibiting prohypertrophic pathways during cardiac hypertrophy can exert an antihypertrophic effect. It was reported that pre-treatment of atorvastatin can prevent the increase in the phosphorylation of Akt and GSK-3 $\beta$ caused by cardiac hypertrophy, and this effect correlated with an increase in protein levels of PTEN, which negatively regulates the phosphoinositide-3 kinase/Akt pathway [9]. Moreover, Planavila et al. elucidated that atorvastatin inhibits cardiac hypertrophy and prevents the decrease in the protein levels of PPAR $\alpha$ and PPAR $\beta / \delta$ and suppresses NF- $\kappa B$ activation during cardiac hypertrophy [27]. In this study, administration of atorvastatin significantly inhibits Ang II-induced cardiomyocytes hypertrophy in vitro and in vivo, which is consistent with previous study. However, the potential mechanisms of these protective effects have not been fully disclosed. In the current study we found that miR-22 is involved in antihypertrophic effect of atorvastatin in vitro and in vivo.

A growing body of evidence shows that specific miRNAs are extensively involved in the pathogenesis of cardiac hypertrophy. For example, miR-9 is involved in regulating a hypertrophic pathway which is constituted of nuclear factor of activated T cells c3 (NFATc3) and myocardin [28]. Dong and his cooperators have domenstrated that reciprocal repression between microRNA-133 and calcineurin regulates cardiac hypertrophy [29]. It has been shown in several recent studies that miR-22 plays a critical role in pathological cardiac hypertrophy. Huang et al. uncovered that miR-22 is a critical regulator of cardiomyocyte hypertrophy and cardiac remodeling [30]. Priyatansh Gurha et al. have demonstrated that miR-22 functions as an integrator of $\mathrm{Ca}^{2+}$ homeostasis and myofibrillar protein content during stress in the miR-22 deficient mouse heart and shed light on the potential mechanisms that enhance propensity toward heart failure [31]. Consistent with these reports, our data showed that the expression level of miR-22 was markedly upregulated in Ang II-induced cardiomyocytes hypertrophy. In addition, pre-treatment of atorvastatin could suppress AngII-induced miR-22 upregulation. As previously reported, miRNAs modulated their biological functions through regulating gene expression post-transcriptionally, by base-pairing to partially complementary sequences in target mRNAs. Using miRNA target predictions (TargetScan, http://www. targetscan.org), we found that PTEN is a potential target of miR-22. Coincidentally, Xu and his cooperators demonstrated that attenuation of miR-22 in neonatal rat cardiomyocytes efficiently protected from hypertrophic effects through derepressing PTEN [21].

Taken together, in this study we disclosed the important role of specific miRNAs in the control of Ang II-induced cardiac hypertrophy and pointed out that miR-22 could be used as potential pharmacological intervention target for the treatment of cardiac hypertrophy. Regulating miRNAs expression by pharmacological treatments are likely to emerge as alternative and safe methods to treat cardiovascular disease. However, it should be aware that our researches were performed in mouse models and the findings may not be extrapolated directly to humans. Additional studies are required to investigate whether the miR-22 mechanism of atorvastatin actions also operate in the clinical setting.

\section{Acknowledgements}

This work was supported by International Cooperation and Exchange of the National Natural Science Foundation of China (Grant No:31210103913); China National Natural Science Funds for Young Scholar (Grant No:81201125).

\section{Conflict of interest}

None declared. 


\section{Cellular Physiology and Biochemistry}

Tu/Wan/Bu/Zhao/Dong/Huang/Cheng/Shen: MicroRNA-22 Downregulation by Atorvastatin in Mouse Cardiac Hypertrophy

\section{References}

1 Bell D, Campbell M, Wang X, Earle JA, Cosby SL, McDermott BJ: Adrenomedullin gene delivery is cardioprotective in a model of chronic nitric oxide deficiency combining pressure overload, oxidative stress and cardiomyocyte hypertrophy. Cell Physiol Biochem 2010;26:383-394.

- Maass AH, Ikeda K, Oberdorf-Maass S, Maier SK, Leinwand LA: Hypertrophy, fibrosis, and sudden cardiac death in response to pathological stimuli in mice with mutations in cardiac troponin t. Circulation 2004;110:2102-2109.

- 3 Senthil V, Chen SN, Tsybouleva N, Halder T, Nagueh SF, Willerson JT, Roberts R, Marian AJ: Prevention of cardiac hypertrophy by atorvastatin in a transgenic rabbit model of human hypertrophic cardiomyopathy. Circ Res 2005;97:285-292.

4 Chiu CZ, Wang BW, Shyu KG: Use of atorvastatin to inhibit hypoxia-induced myocardin expression. Eur J Clin Invest 2012;42:564-571.

-5 Laufs U, Kilter H, Konkol C, Wassmann S, Bohm M, Nickenig G: Impact of hmg coa reductase inhibition on small gtpases in the heart. Cardiovasc Res 2002;53:911-920.

6 Rodrigues Diez R, Rodrigues-Diez R, Lavoz C, Rayego-Mateos S, Civantos E, Rodriguez-Vita J, Mezzano S, Ortiz A, Egido J, Ruiz-Ortega M: Statins inhibit angiotensin ii/smad pathway and related vascular fibrosis, by a tgf-beta-independent process. PLoS One 2010;5:e14145.

7 Crackower MA, Oudit GY, Kozieradzki I, Sarao R, Sun H, Sasaki T, Hirsch E, Suzuki A, Shioi T, Irie-Sasaki J, Sah R, Cheng HY, Rybin VO, Lembo G, Fratta L, Oliveira-dos-Santos AJ, Benovic JL, Kahn CR, Izumo S, Steinberg SF, Wymann MP, Backx PH, Penninger JM: Regulation of myocardial contractility and cell size by distinct pi3k-pten signaling pathways. Cell 2002;110:737-749.

-8 Schwartzbauer G, Robbins J: The tumor suppressor gene pten can regulate cardiac hypertrophy and survival. J Biol Chem 2001;276:35786-35793.

-9 Planavila A, Rodriguez-Calvo R, Palomer X, Coll T, Sanchez RM, Merlos M, Laguna JC, Vazquez-Carrera M: Atorvastatin inhibits gsk-3beta phosphorylation by cardiac hypertrophic stimuli. Biochim Biophys Acta 2008;1781:26-35.

10 Bostjancic E, Zidar N, Stajer D, Glavac D: Micrornas mir-1, mir-133a, mir-133b and mir-208 are dysregulated in human myocardial infarction. Cardiology 2010;115:163-169.

11 Zhang Y, Zhang L, Chu W, Wang B, Zhang J, Zhao M, Li X, Li B, Lu Y, Yang B, Shan H: Tanshinone iia inhibits mir-1 expression through p38 mapk signal pathway in post-infarction rat cardiomyocytes. Cell Physiol Biochem 2010;26:991-998.

12 Fiedler J, Jazbutyte V, Kirchmaier BC, Gupta SK, Lorenzen J, Hartmann D, Galuppo P, Kneitz S, Pena JT, SohnLee C, Loyer X, Soutschek J, Brand T, Tuschl T, Heineke J, Martin U, Schulte-Merker S, Ertl G, Engelhardt $\mathrm{S}$, Bauersachs J, Thum T: Microrna-24 regulates vascularity after myocardial infarction. Circulation 2011;124:720-730.

13 Zhang L, Wu Y, Li Y, Xu C, Li X, Zhu D, Zhang Y, Xing S, Wang H, Zhang Z, Shan H: Tanshinone iia improves mir-133 expression through mapk erk1/2 pathway in hypoxic cardiac myocytes. Cell Physiol Biochem 2012;30:843-852.

14 Lu Y, Zhang Y, Wang N, Pan Z, Gao X, Zhang F, Shan H, Luo X, Bai Y, Sun L, Song W, Xu C, Wang Z, Yang B: Microrna-328 contributes to adverse electrical remodeling in atrial fibrillation. Circulation 2010;122:2378-2387.

15 Wei C, Kim IK, Kumar S, Jayasinghe S, Hong N, Catalucci D, Castoldi G, Jones WK, Gupta S: Nf-kappab mediated mir-26a regulation in cardiac fibrosis. J Cell Physiol 2012. doi: 10.1002/jcp.24296.

16 Kumarswamy R, Anker SD, Thum T: Micrornas as circulating biomarkers for heart failure: Questions about mir-423-5p. Circ Res 2010;106:e8; author reply e9.

-17 Blanco RR, Austin H, Vest RN 3rd, Valadri R, Li W, Lassegue B, Song Q, London B, Dudley SC, Bloom HL, Searles CD, Zafari AM: Angiotensin receptor type 1 single nucleotide polymorphism $1166 \mathrm{a} / \mathrm{c}$ is associated with malignant arrhythmias and altered circulating mir-155 levels in patients with chronic heart failure. J Card Fail 2012;18:717-723.

18 Han M, Yang Z, Sayed D, He M, Gao S, Lin L, Yoon S, Abdellatif M: Gata4 expression is primarily regulated via a mir-26b-dependent post-transcriptional mechanism during cardiac hypertrophy. Cardiovasc Res 2012;93:645-654. 


\section{Cellular Physiology and Biochemistry}

19 Wang J, Song Y, Zhang Y, Xiao H, Sun Q, Hou N, Guo S, Wang Y, Fan K, Zhan D, Zha L, Cao Y, Li Z, Cheng X, Yang $\mathrm{X}$ : Cardiomyocyte overexpression of mir-27b induces cardiac hypertrophy and dysfunction in mice. Cell Res 2012;22:516-527.

20 Roy S, Khanna S, Hussain SR, Biswas S, Azad A, Rink C, Gnyawali S, Shilo S, Nuovo GJ, Sen CK: Microrna expression in response to murine myocardial infarction: Mir-21 regulates fibroblast metalloprotease-2 via phosphatase and tensin homologue. Cardiovasc Res 2009;82:21-29.

21 Xu XD, Song XW, Li Q Wang GK, Jing Q, Qin YW: Attenuation of microrna-22 derepressed pten to effectively protect rat cardiomyocytes from hypertrophy. J Cell Physiol 2012;227:1391-1398.

-22 Brock M, Samillan VJ, Trenkmann M, Schwarzwald C, Ulrich S, Gay RE, Gassmann M, Ostergaard L, Gay S, Speich R, Huber LC: Antagomir directed against mir-20a restores functional bmpr2 signalling and prevents vascular remodelling in hypoxia-induced pulmonary hypertension. Eur Heart J 2012, epub ahead of print.

23 Uramoto H, Okada T, Okada Y: Protective role of cardiac cftr activation upon early reperfusion against myocardial infarction. Cell Physiol Biochem 2012;30:1023-1038.

24 Oudit GY, Kassiri Z, Zhou J, Liu QC, Liu PP, Backx PH, Dawood F, Crackower MA, Scholey JW, Penninger JM: Loss of pten attenuates the development of pathological hypertrophy and heart failure in response to biomechanical stress. Cardiovasc Res 2008;78:505-514.

25 Feng Y, Hans C, McIlwain E, Varner KJ, Lazartigues E: Angiotensin-converting enzyme 2 over-expression in the central nervous system reduces angiotensin-ii-mediated cardiac hypertrophy. PLoS One 2012; 7:e48910.

26 Lin Z, Murtaza I, Wang K, Jiao J, Gao J, Li PF: Mir-23a functions downstream of nfatc3 to regulate cardiac hypertrophy. Proc Natl Acad Sci U S A 2009;106:12103-12108.

27 Planavila A, Laguna JC, Vazquez-Carrera M: Atorvastatin improves peroxisome proliferator-activated receptor signaling in cardiac hypertrophy by preventing nuclear factor-kappa b activation. Biochim Biophys Acta 2005;1687:76-83.

28 Wang K, Long B, Zhou J, Li PF: Mir-9 and nfatc3 regulate myocardin in cardiac hypertrophy. J Biol Chem 2010;285:11903-11912.

29 Dong DL, Chen C, Huo R, Wang N, Li Z, Tu YJ, Hu JT, Chu X, Huang W, Yang BF: Reciprocal repression between microrna-133 and calcineurin regulates cardiac hypertrophy: A novel mechanism for progressive cardiac hypertrophy. Hypertension 2010;55:946-952.

30 Huang ZP, Chen J, Seok HY, Zhang Z, Kataoka M, Hu X, Wang DZ: Microrna-22 regulates cardiac hypertrophy and remodeling in response to stress. Circ Res 2013;112:1234-1243.

-31 Gurha P, Abreu-Goodger C, Wang T, Ramirez MO, Drumond AL, van Dongen S, Chen Y, Bartonicek N, Enright AJ, Lee B, Kelm RJ Jr, Reddy AK, Taffet GE, Bradley A, Wehrens XH, Entman ML, Rodriguez A: Targeted deletion of microrna-22 promotes stress-induced cardiac dilation and contractile dysfunction. Circulation 2012;125:2751-2761. 